\title{
Dentadura completa en pacientes con paladar hendido
}

\author{
Full denture in patient with cleft palate
}

\section{Resumen}

Reportamos la rehabilitación de un paciente edentulo total que presenta paladar hendido. Por tratarse de un caso complejo que afecta a uno de los factores de la estabilidad (retención), se tuvo que utilizar materiales de mayor hidrofilicidad para tomar las impresiones. Se usó la técnica sin presión para la impresión primaria y la técnica de presión selectiva (elastómero) para la toma de la impresión definitiva; obteniendo así mayor intimidad de contacto entre la prótesis y la mucosa, para mejor adhesión y cohesión. Se utilizaron cubetas completamente aliviadas para que la presión del material de impresión sea mínima. El propósito de este tratamiento fue rehabilitar el sistema masticatorio del paciente edentulo total con paladar hendido, que a diferencia de los casos comunes, la adaptación de la prótesis a la mucosa debe ser íntima; manteniendo la resilencia de la mucosa y permitiendo así una buena retención.

Palabras clave: Prótesis dental, paladar hendido.

\begin{abstract}
We report the rehabilitation of a total edentulous patient with cleft palate. Being a complex case involving one of the factors of stability (retention), we had to use materials with grater hydrophilicity to take impressions. The technique without pressure was used for primary impressions and technique of selective pressure (elastomer) for making the final impression; obtaining more intimate contact between the prosthesis and the mucosa, for better adhesion and cohesion. Completely relieved cuvettes were used to take impressions with a minimal pressure.

The purpose of this treatment was to rehabilitate the masticatory system of total edentulous patient with cleft palate, unlike ordinary cases, the adaptation of the prosthesis to the mucosa should be intimate; maintaining the resilience of the mucosa and allowing good retention.

Keywords: Dental prosthesis, cleft palate.
\end{abstract}

\section{Introducción}

La rehabilitación de un paciente edéntulo total con una reabsorción extrema del reborde alveolar es un desafío afrontado por los especialistas en rehabilitación oral ${ }^{1}$.

Los aspectos biológicos son un factor importante para un buen pronóstico en el tratamiento de un paciente desdentado total. ${ }^{2}$ Los defectos óseos en el área del tejido de soporte tanto en el maxilar superior como en el inferior son muy comunes, los cuales nos llevan frecuentemente a una preparación preprotésica. ${ }^{3-4}$

La altura del reborde alveolar es muy importante puesto que dará buen soporte a la dentadura completa y resistirá mejor los movimientos laterales. ${ }^{5-6}$ Así como también la forma del paladar duro ayuda a la retención y soporte vertical de la prótesis superior. ${ }^{7-8}$

Los pacientes con defectos congénitos en los maxilares, como es el caso del paciente con fisura palatina, presenta defectos físicos en la geometría del reborde y el paladar; que nos complican en el desarrollo de los objetivos del tratamiento, los cuales son la retención y la estabilidad de la prótesis en la boca de nuestros pacientes. ${ }^{9-10}$

La inestabilidad de la prótesis es el segundo problema que con frecuencia encontramos en comparación con el dolor que presentan los pacientes con dentaduras completas post inserción. ${ }^{11}$

Siempre que tengamos un caso complicado y busquemos la estabilidad de la prótesis, tenemos que ser prolijos en los procedimientos clínicos y de laboratorio, considerando cuatro aspectos importantes para la estabilidad de la prótesis como son: ${ }^{12-13}$

- Soporte adecuado

- Buena retención

- Buen equilibrio muscular

- Equilibrio oclusal adecuado

\section{Casos Clínicos}

\author{
Ernesto Vilchez Salazar ${ }^{1}$ \\ Waldo Gloria Zeballos ${ }^{1}$ \\ Ernesto Vilchez Quintana ${ }^{2}$ \\ Carol Torres Roque ${ }^{2}$
}

1 Departamento Académico de Estomatología Rehabilitadora, Facultad de Odontología, Universidad Nacional Mayor de San Marcos. Perú.

2 Bachiller de odontología, Facultad de Odontología, Universidad Nacional Mayor de San Marcos. Perú.

\section{Correspondencia:}

Mg. Esp. Ernesto Vílchez Salazar.

Facultad de Odontología. Universidad Nacional Mayor de San Marcos. Av. Germán Amézaga s/n. Lima 1. Perú.

Correo electrónico: ernyrex@hotmail.com

Fecha de recepción: 07-05-13

Fecha de aceptación: 19-06-14

La estabilidad es el factor que define el éxito de una dentadura completa considerando también todos los aspectos antes mencionados.

En los pacientes con defectos en los rebordes residuales y rebordes completa-mente reabsorbidos, hay que tener mucho cuidado al momento de la toma de impresiones, ya que este paso va a ser de vital importancia para obtener la estabilidad de la prótesis.

La forma y tamaño de los rebordes influyen directamente en la distribución de fuerzas en la rehabilitación de un paciente edéntulo total.

Los dos procesos palatinos de los maxilares y del hueso palatino forman la estructura del paladar duro y proporcionan un soporte considerable para la dentadura total. Por ello el reborde residual y la mayor parte del paladar duro son considerados como el área más importante para el soporte de tención en el maxilar.

La cresta del reborde alveolar residual está cubierta por una capa de tejido conectivo fi- 
broso, que es muy favorable para soportar la dentadura por su firmeza y posición.

Los tejidos de soporte de un paciente que presenta paladar fisurado, son altamente resilientes principalmente en el sector anterior. Si se presiona en un punto se deforma la mucosa, pero lo que sucede realmente es el desplazamiento de los líquidos de este punto hacia las zonas vecinas; al retirar la presión, estos líquidos vuelven a recuperar su posición anterior y la mucosa adquiere su forma original. A esta propiedad de desplazamiento de líquidos y recuperación de la forma se llama resiliencia de la mucosa.

Si se toma una impresión de la mucosa, principalmente en zonas altamente resilientes, se tiene que utilizar un material de impresión que no comprima los tejidos (como silicona de adición) y el resultado será la máxima adaptación de la dentadura a los tejidos de soporte. Por otro lado, si se usa un material de impresión de tipo pesado deformará la resiliencia de la mucosa, perdiéndose así la adaptación de la base de la dentadura a los tejidos, con lo que desaparecerá el principal factor de la retención.

La técnica por presión selectiva es muy utilizada para los casos con resorción ósea extrema la cual presenta dos fases de impresión, la primera es la fase del sellado periférico (con compuesto de modelar de baja fusión) y la segunda es la que utiliza un material más fluido (como por ejemplo la silicona).

La superficie de oclusión en la prótesis total es mucho más importante en pacientes con defectos de los rebordes residuales ya que las fuerzas oclusales equilibradas o balanceadas van a contribuir en la estabilidad de la prótesis total en la boca del paciente.

\section{Caso clínico}

Paciente de sexo masculino de 56 años de edad que acude a la Clínica Odontológica de Pre-grado de la Universidad Nacional Mayor de San Marcos presentando ausencia total de piezas, paladar fisurado, asimetría labial, cicatriz labial unilateral izquierda. Radiográficamente se observa ausencia de consolidación de los procesos maxilares. (Fig. 1 y 2)

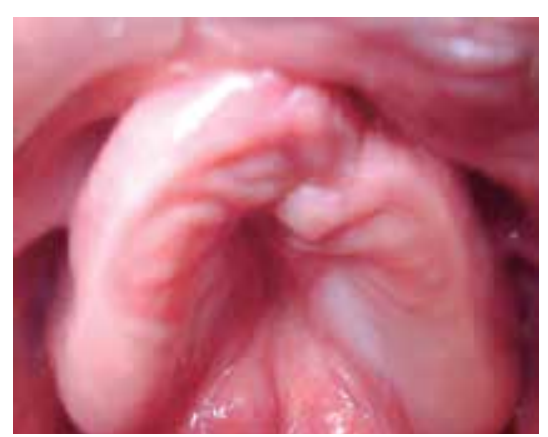

Fig. 1 Fotografía intraoral de la arcada superior.

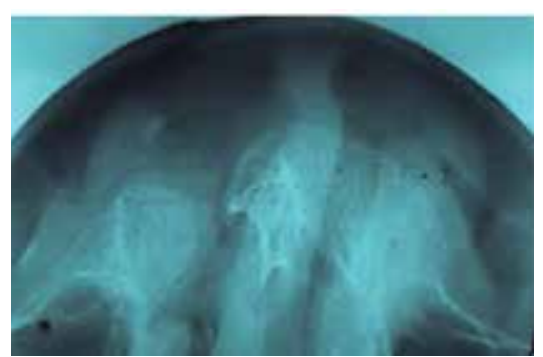

Fig. 2 Radiografía oclusal de paladar superior fisurado.

\section{Diagnóstico}

Paciente edéntulo total con paladar fisurado, buen estado de salud general según la clasificación de House es un paciente filosófico.

\section{Tratamiento}

Se tomó la impresión primaria, con la técnica sin presión, utilizando una cubeta completamente espaciada y cribada ya que se quería mantener la resiliencia de la mucosa, principalmente de la zona de la fisura palatina y de esta manera obtener una copia fiel y exacta para la obtención de los modelos primarios, los cuales sirvieron para la confección de las cubetas individuales. (Fig 3).

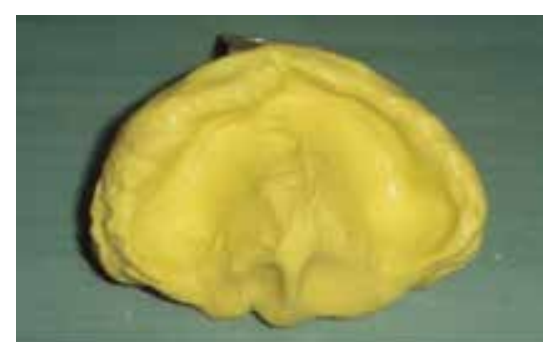

Fig. 3 Impresión primaria utilizando la técnica sin presión con cubeta completamente espaciada.

Estas cubetas fueron debidamente diseñadas y completamente aliviadas especialmente en las zonas de la fisura palatina, luego fueron utilizadas en la toma de impresión definitiva con la técnica de presión selectiva, con esta técnica lo que se quiere obtener era una buena retención considerando los tres factores principales de la retención los cuales son: Extensión de la base, intimidad de contacto y sellado periférico. (Fig. 4).

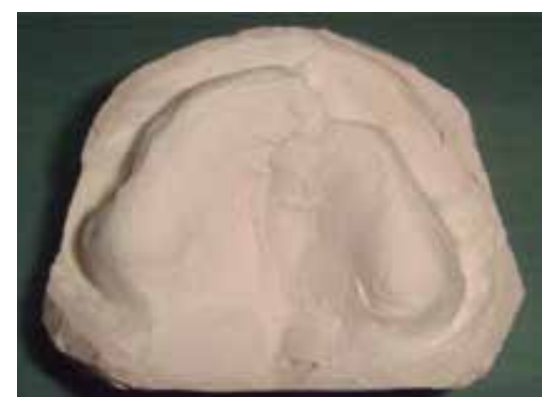

Fig. 4 Modelo de trabajo obtenido de la técnica de impresión por presión selectiva
Para la confección de la placa base del presente caso clínico, se tuvo que aliviar la zona de la fisura palatina para evitar la fractura del modelo en el momento de retirar la base de registro.

Para éste caso, fue muy importante la toma de un buen registro intermaxilar, para ello se utilizaron las bases de registro bien adaptadas en boca y el arco facial. (Fig. 5)

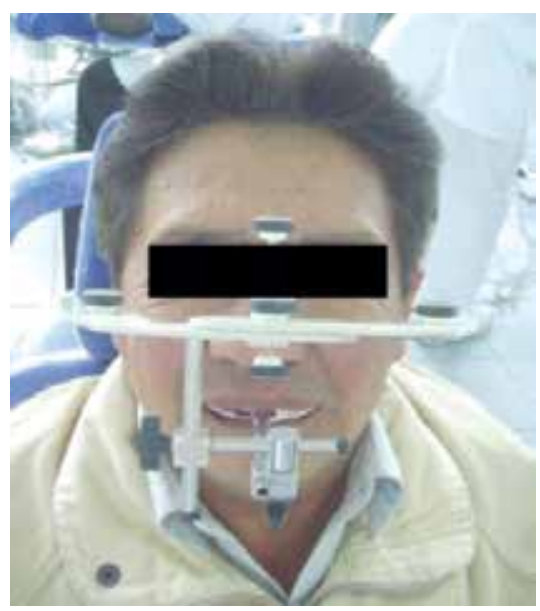

Fig. 5 Toma de registro con el arco facial.

Se utilizó el esquema oclusal de tipo monoplano ya que las fuerzas horizontales son menores y de esta manera contribuye a la estabilidad de la prótesis. (Fig. 6)

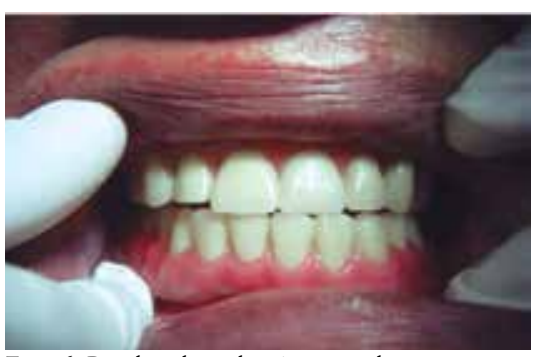

Fig. 6 Prueba de oclusión con dientes monoplano.

En estos casos clínicos es muy importante la oclusión balanceada ya que las fuerzas transmitidas por los músculos hacia la superficie oclusal de las piezas artificiales deben estar equilibradas, para lo cual utilizamos el papel artificial. (Fig. 7)

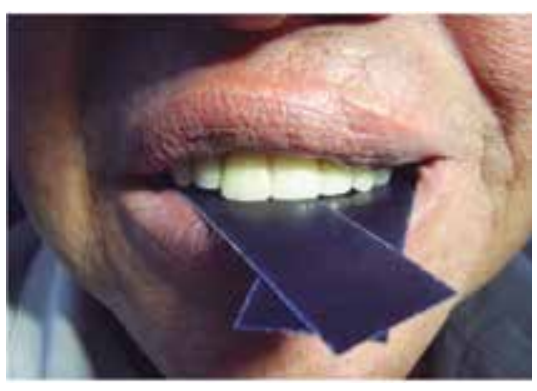

Fig. 7.Balance oclusal con el papel articular 


\section{Resultados}

Los resultados fueron satisfactorios, ya que se logró devolver la estética, la función y el aspecto psicológico del paciente puesto que tras haber ido a distintos lugares, ya se encontraba resignado a quedarse sin la confección de su prótesis.

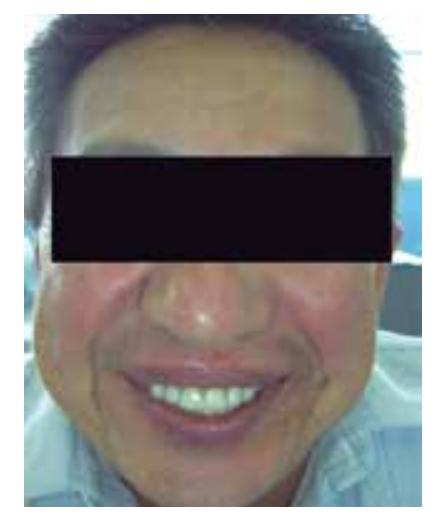

Fig. 8.Caso clínico terminado.

\section{Discusión}

El tratamiento de los pacientes edéntulos totales, es una de las intervenciones clínicas más difíciles en odontología. La elevada frecuencia de complicaciones que diversos autores cifran entre el 50 y 90 $\%$, condiciona que la reconstrucción de la forma y la función de los dientes sea un modo de tratamiento.

Las investigaciones de Plasencia (2001) ${ }^{11}$ han puesto de manifiesto que la retención de la prótesis es directamente proporcional al área de soporte; por ello en el caso del paladar fisurado se tendría poca área de soporte.

Los procesos que ocasiona la mala formación en las crestas alveolares es consecuencia directa de la pérdida ósea, que conlleva a una disminución constante de la disminución vertical con reducción de la altura facial. Winkler $(1990)^{1}$ descubrió que los pacientes con defectos congénitos, como es del caso con paladar fisurado de nuestro paciente, tienen la mucosa pendular que dificulta la impresión por lo cual no tendremos una buena adaptación.

En caso de pacientes con defectos en el reborde alveolar, como el presente caso clínico, los objetivos y condiciones de la primera y segunda impresión deben considerarse siempre en conjunto. Se trata de procedimientos dependientes entre sí, lo que conllevó a la utilización de un método de impresión para la impresión primaria y otro método para la impresión definitiva. Por tal razón se dice que la toma de impresión es un parámetro esencial para conseguir una buena retención de la prótesis.

\section{Conclusiones}

Para responder la exigencia de este caso el clínico debe tener un juicio clínico para establecer un plan de tratamiento correcto y así llegar a la solución protésica precisa. Es importante ser prolijo en la elección de la técnica a utilizar en un determinado caso clínico. En este caso se tuvo que utilizar dientes artificiales con esquema oclusal monoplano, puesto que el paciente presentó rebordes no muy uniformes en donde hay que disminuir las fuerzas horizontales que pueden llevar a desestabilizar la prótesis. La técnica de impresión definitiva con material de mayor hidrofilicidad (silicona de adición) nos produce mejor contacto de superficie entre la base de la prótesis y la mucosa, de esta manera se creará mayor retención de la prótesis.

Las impresiones no deben modificar la resiliencia de la mucosa, es decir, las impresiones deben producir la mínima presión posible.

En tipos de tratamiento rehabilitador hay que relacionar tres aspectos fundamentales: el equilibrio oclusal, equilibrio muscular y retención.

\section{Referencias Bibliográficas}

1. Winkler S. Prostodoncia Total. $2^{\circ}$ edi. México: Interamericana. 1990. 401461p.

2. Boucher C. Prostodoncia Total. $1^{\circ}$ ed. México: Interamericana. 1998. 230238p.

3. Feltrin P. Estudio Clínico de la reabsorción ósea en pacientes portadores de Prótesis Total. $2^{\circ}$ ed. Brasil: Interamericana. 2001. 230238p.

4. Kawabe A. Dentaduras Totales $1^{\circ}$ ed. Japón: Interamericana. 2001. 80-87p.

5. Alfred H. Prótesis Total y Sobredentaduras. $1^{\circ}$ ed. España. Interamericana. 2001. 144-118p.

6. Korholz H. Prótesis Total en función. 1ra. ed. Editorial Española. 2000. 150$160 \mathrm{p}$.

7. Jengan S. Errores Comunes en las dentaduras Completas. Quintessence 1995;8(4):55-61.

8. Capuselli H. Tratamiento del desdentado total, $2^{\circ}$ ed. México: Ed. Mundi. 1980. 201-221p.

9. Tortamano N. Estudio de superficie interna de la Prótesis Total en microscopia electrónica de barrido en mucosa de soporte a través del examen citológico, histopatológico e inmuno histoquímico. CEFAC. 2001; 49(1):27-32.

10. Bernald B; Moreira D. La respuesta inflamatoria celular en la estomatitis subprotésica. Rev. Cubana de Estomatología 1995;15(2):161-167.

11. Plasencia J. Prótesis Completa. $1^{\circ}$ ed. España: Interamericana. 2001. 65$71 \mathrm{p}$.

12. Harinasuta S. Keratinization of palatal mucosa beneath metalbased removable partial dentures. J Dent. 1992 Jun;20(3):152-5.

13. Koeck B. Prótesis Completas. $1^{\circ}$ ed. España: Interamericana. 2001:81-85. 